\title{
Téoros
}

Revue de recherche en tourisme

\section{La question du statut scientifique du tourisme}

\section{Boualem Kadri}

Volume 27, numéro 1, printemps 2008

Science du tourisme ou études touristiques?

URI : https://id.erudit.org/iderudit/1070889ar

DOI : https://doi.org/10.7202/1070889ar

Aller au sommaire du numéro

Éditeur(s)

Université du Québec à Montréal

ISSN

0712-8657 (imprimé)

1923-2705 (numérique)

Découvrir la revue

Citer ce document

Kadri, B. (2008). La question du statut scientifique du tourisme. Téoros, 27(1),

5-6. https://doi.org/10.7202/1070889ar d'utilisation que vous pouvez consulter en ligne.

https://apropos.erudit.org/fr/usagers/politique-dutilisation/ 


\section{La question du statut scientifique du tourisme}

\section{Boualem Kadri}

Depuis plus d'une décennie, le tourisme s'affirme comme un élément important de l'économie des services et un outil stratégique d'appui au développement, mais il peine toutefois à construire une reconnaissance scientifique et une identité propre. Pourtant, depuis un demi-siècle, la pensée scientifique en tourisme a évolué, passant d'une réflexion centrée sur une accusation du tourisme à un intérêt à développer un corps de connaissances. Cet effort dans l'étude du phénomène renforce certes la reconnaissance scientifique du tourisme (programmes de formation supérieure, revues et journaux spécialisés, organisations et associations internationales, rencontres scientifiques internationales), mais il met aussi en évidence deux handicaps importants : 1) une fragmentation dans la recherche en tourisme (diversité de disciplines et d'acteurs) ; 2) une absence de consensus autour d'une définition "académique » du tourisme et de son statut scientifique (discipline? champ d'études ? science autonome?). Les positions concernant ce statut divisent la communauté des chercheurs: il y a, d'un côté, ceux qui estiment que le tourisme a besoin d'un statut disciplinaire et scientifique distinct et, de l'autre, ceux qui considèrent que le tourisme est un champ d'études qui n'a pas l'envergure épistémologique d'une discipline et d'une science. Entre ces deux pôles, il y a ceux qui restent convaincus de la priorité à accorder à la construction d'un corps de connaissances comme préalable à la création d'une science nouvelle, celle du tourisme. Cette permanence des problèmes liés aux aspects épistémologiques et aux luttes entre disciplines et intervenants en tourisme peut trancher avec certaines propositions d'acteurs politiques visant le renforcement d'une reconnaissance de la discipline. Ce fut le cas en France, en 2006,

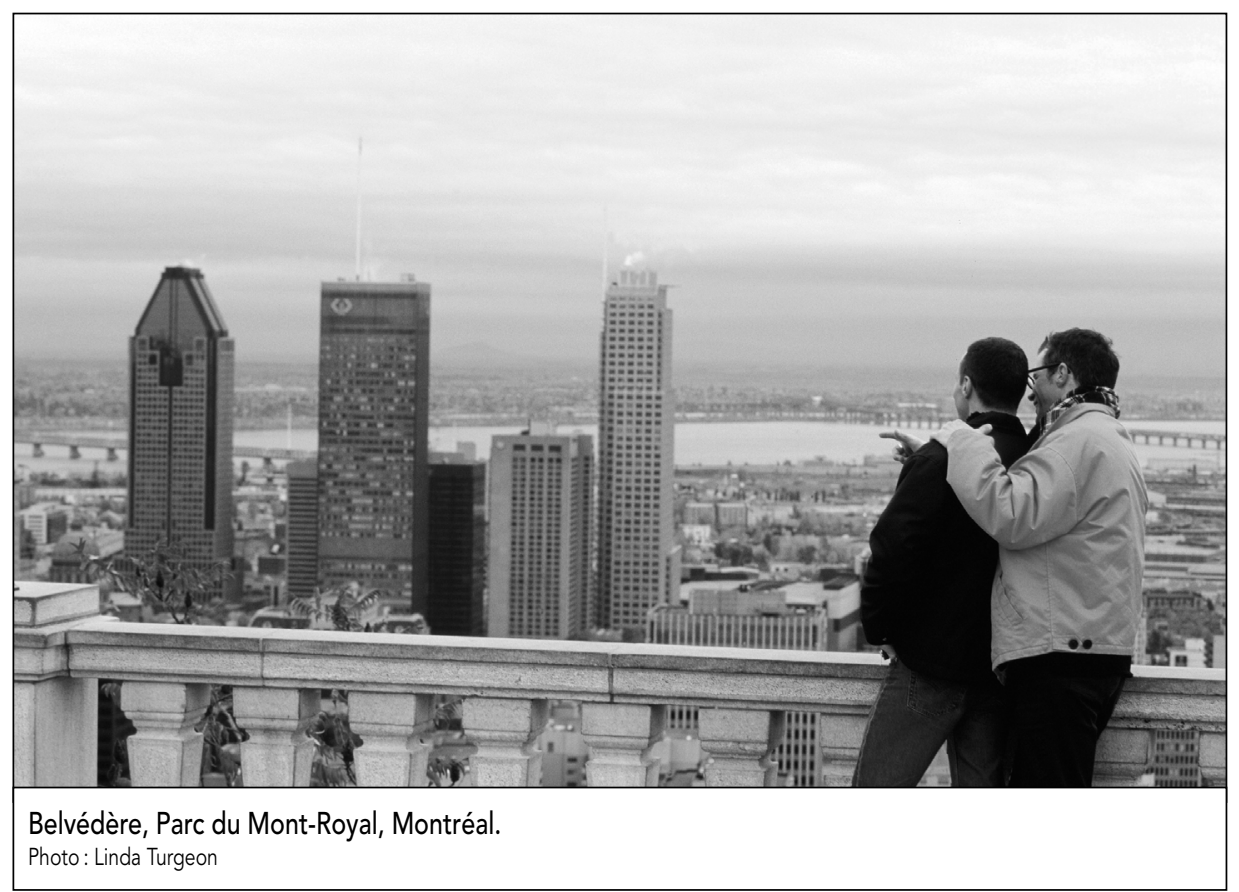

où l'on a avancé, par exemple, l'idée de créer une "agrégation en tourisme ». Toutefois, la reconnaissance politique ne peut ni remplacer la reconnaissance scientifique ni faire l'économie d'un débat et d'une recherche de consensus dans la communauté scientifique.

La revue de recherche en tourisme Téoros veut renouer avec la réflexion scientifique sur le tourisme, déjà entamée dans les années 1980-1990, et participer au débat qui entoure la constitution d'une science du tourisme. Un tel débat a commencé en France, en 2000, dans la revue Espaces, mais il a été marqué surtout par les tensions entre les tenants d'une même discipline (la géographie) et entre les pôles de la recherche en tourisme (l'université et les associations d'experts en tourisme). Précisons, néanmoins, que ce débat ne se déroule pas exclusivement sur le terrain de la recherche francophone; il a lieu aussi entre chercheurs anglo-saxons, par l'intermédiaire de la revue Annals of Tourism Research.

Avec ce numéro thématique intitulé "Science du tourisme ou études touristiques ? ", questionnement qui par ailleurs ne cherche nullement à enfermer la solution identitaire du tourisme dans un choix à deux pôles, Téoros souhaite bien au contraire promouvoir un débat organisé dans une perspective internationale et pluridisciplinaire. L'objectif de ce numéro est de s'interroger à nouveau sur l'identité scientifique du tourisme, en tentant de mettre en évidence les handicaps épistémologiques et méthodologiques, de comprendre les raisons qui freinent la construction d'un consensus au sein de la communauté scientifique, d'identifier les conditions nécessaires à l'émergence d'une science du tourisme aujourd'hui. 


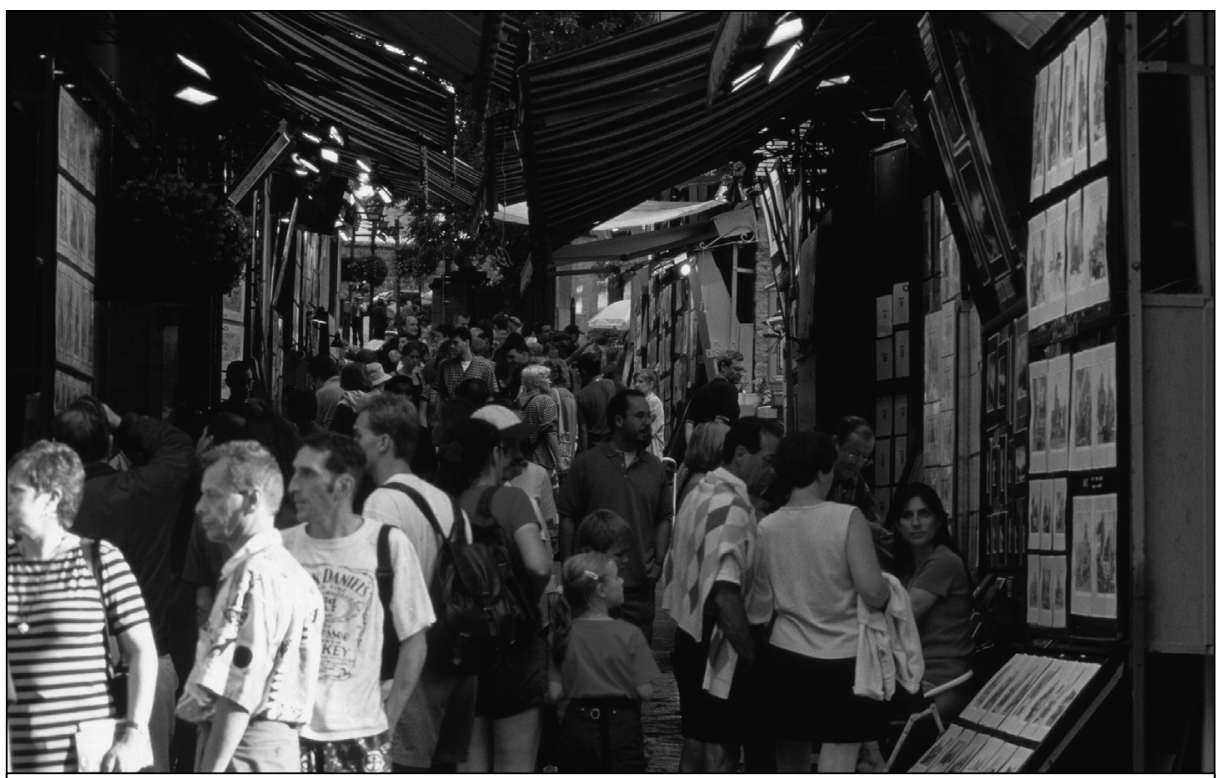

Rue du Trésor, Vieux-Québec, Québec.

Photo : Linda Turgeon

Pour Giorgia Ceriani-Sebregondi, Amandine Chapuis, Jean-Christophe Gay, Rémy Knafou, Mathis Stock et Philippe Violier, il y a des étapes à franchir pour définir le statut scientifique du tourisme, notamment : constituer des concepts et des méthodologies propres, ramener le touriste au centre de la préoccupation scientifique et non le discréditer, et considérer les tourism studies comme une étape importante du débat interdisciplinaire et comme préalable à la naissance d'une science du tourisme.

Jean-Michel Hoerner, qui a été à l'origine de la relance du débat sur la science du tourisme en France, en 2000, milite pour la création d'une science, ici et maintenant, qui aura un caractère pluridisciplinaire et sera centrée sur les réalités économiques du tourisme, impliquant alors une révision de certaines notions véhiculées par l'Organisation mondiale du tourisme: par exemple celle de " touriste", comprise actuellement dans sa dimension de la durée, serait à relier plutôt à celle de la consommation touristique.

Le chemin vers l'autonomie scientifique est semé d'obstacles. Néanmoins, comme le rappelle Gilles Pronovost, la quête d'une légitimité scientifique du tourisme s'est construite sur des emprunts conceptuels extérieurs (à partir des sciences du loisir, de la géographie, de l'anthropologie, de la psychologie, etc.), induisant alors une fragmentation dans la recherche. Pour cet auteur, l'intention de fonder à nouveau une légitimité scienti- fique sur un paradigme unique serait vaine, alors que la démarche d'une interdisciplinarité serait davantage prometteuse.

Jean-Michel Dewailly également montre l'intérêt d'une collaboration entre les disciplines, d'autant que la complexité du phénomène étudié bouscule les frontières disciplinaires, nécessitant à la fois une approche transdisciplinaire et un apport des théories de la complexité et du chaos qui permettent une meilleure compréhension du phénomène touristique.

La reconnaissance scientifique du tourisme passe en outre par l'importance et la qualité de la formation supérieure. À ce propos, David Airey analyse les diverses étapes qui ont conduit, depuis les années 1960, à l'émergence du tourisme comme champ d'études et domaine de la formation supérieure et il évalue l'état d'une possible «maturité » de celui-ci (40 ans, déjà!), ce qui contribuerait fortement à la reconnaissance scientifique du tourisme.

L'importance de la formation supérieure en tourisme est relevée aussi par Brent Ritchie, Seldjan Timur et Lorn Sheehan. Évaluant le poids de la dimension des fondements théoriques (tourism sciences) et celui de l'application (tourism studies) dans la formation supérieure en management du tourisme, ces auteurs construisent une perspective à la fois théorique et managériale: ce rapprochement entre la théorie (fondement théorique du tourisme) et la pratique (activité managériale) prend forme, selon eux, dans le cadre du modèle global incarné par «la destination ", où le touriste retrouve sa place centrale.

Par ce dernier aspect, la réflexion managériale en tourisme rejoint l'approche géographique. Le dialogue entre disciplines est donc possible et un consensus scientifique peut en émerger.

Au-delà des problèmes épistémologiques et méthodologiques, il y a les intentions des divers intervenants. À ce propos, Mimoun Hillali se penche sur les discours entourant le projet de création d'une science du tourisme, depuis 2000, en France; il analyse les positions et les motivations des divers acteurs en présence (les autonomistes, les opposants, les neutres) et s'interroge : "débat ou polémique? ".

Problèmes épistémologiques, luttes inter- et intradisciplinaires, complexité du phénomène nécessitant de nouveaux outils d'analyse, etc. présentent les contours d'une identité qui a des difficultés à se définir. Le tourisme a-t-il un avenir en tant que science et discipline? Quelle épistémologie envisager face aux critères positivistes qui déclarent ce qui est scientifique ou ne l'est pas? L'enjeu est donc aussi de revoir la pertinence épistémologique d'évaluation d'une science, d'une discipline dans les sciences sociales. Boualem Kadri propose, d'une part, de s'intéresser à la perspective offerte par l'épistémologie interne et constructiviste qui se réalise au sein des sciences sociales et qui permet la construction de leur identité scientifique et, d'autre part, de considérer l'exemple de la construction épistémologique qui a lieu dans les sciences de la gestion comme pouvant éclairer l'effort à consentir en tourisme.

Nous espérons que ce numéro thématique de Téoros consacré à la réflexion épistémologique en tourisme permettra de poursuivre le débat sur son statut scientifique et d'envisager la construction d'un consensus, tout d'abord autour des grandes problématiques d'études du phénomène touristique.

Boualem Kadri est professeur associé au Département d'études urbaines et touristiques de l'Université du Québec à Montréal. 